\title{
Colecistitis aguda, Empiema vesicular y Trabajo de Parto
}

\author{
PRESENTACION DE TRES CASOS \\ Dr. Luis Laverde Mercado
}

Hojeando la literatura obstétrica universal, es muy poco lo que se encuentra respecto a la conducta a seguir en la presencia simultánea de colecistitis aguda o del empiema vesicular, con el embarazo a término y trabajo de parto.

\section{FRECUENCIA}

Revisando las estadísticas de nuestros grandes servicios obstétricos (Instituto Materno Infantil, Clínicas del Seguro Social, Servicio de familiares de las Fuerzas Armadas, Hospital San Rafael en San Bonifacio de Ibagué) en diez años para cada uno encontramos :

Para el Instituto Materno Infantil $0.208 \%$.

Para el I.C.S.S. 0,456\%.

Consultorio Familiares Fuerzas Armadas, 0,785\%.

Hospital San Rafael de Ibagué, 0,788\%.

Contrariamente a lo que comentan los autores sobre la apendicitis en la gestación, sí predispone entre nosotros el embarazo a las colangiopatías.

$\mathrm{Al}$ respecto se encuentran todas las frecuencias, desde una paciente (Liverde Mercado) a quien se le han practicado siete cesáreas, hallándose en todas el colecisto perfectamente fláccido y lleno de bilis, sin la menor huella calculosa, hasta otras en que se presenta el ataque de angiocolitis en cuanto falta la primera regia. 
No quieren decir estas frases que forzosamente haya agravación de la enfermedad, sino que en general cuando una mujer encinta tiene algo en su vesícula biliar, padece de ordinario de un malestar, de un fastidio orgánico que se podría llamar característico y que traduce un sufrimiento peritoneal: hay una hipersensibilidad difusa, no propiamente dolorosa, sino una sensación de llenura, de incomodidad que se exacerba hacia el hipocondrio derecho.

Dice Bar (4) "Las gestantes tienen un hígado grande y Ennuyeux en un 95\% de los casos. De aquí la subictericia, la pigmentación de la piel y de las cicatrices, la constipación que puede alternar con diarrea biliosa mejorando después de gastralgia y de expulsión de lodo biliar".

Cuál es la causa básica que provoca con excesiva frecuencia o con tanta constancia la presencia de la colecistitis aguda en el embarazo?

Desde 1862 Villemin de Vichy y Durand Fanel la notan. Señalan la enfermedad en un $70 \%$ en las mujeres, cualesquiera que sea su estado, al paso que en el sexo masculino sólo aparece en un $30 \%$. En estos aparece tardíamente. Es en el sexo femenino excepcional en las vírgenes, no rara en las primíparas, frecuente en las multigestantes.

Huchard le atribuye a la gestación una reactivación de la diatesis artrítica; Cyr desde 1861 hablaba de compresión de las vías biliares o de espesamiento de la bilis. Manning tiene un errado concepto de infección vesicular.

En realidad las causas son múltiples: las mecánicas, por compresión hepática en las últimas semanas; biológicas como la colibacilosis gravídica, tan frecuente, tan discreta a veces, pero que tiene un papel indiscutible en la precipitación de la colesterina, origen de los cálculos.

Audebert y Gilles, investigando, encontraron en el $90 \%$ de las grávidas una colesterinemia de 2 gr. o 2,5 gr. o 2,7 grs. contra 1,70 de la mujer no gestante. 
La colesterinemia tiene un doble origen: la suprarrenal, de alto funcionamiento o hiperfuncionamiento permanente en la gestante, traducida histológicamente por una intensa sobrecarga de lipoides, el segundo la hiperfunción del cuerpo amarillo, de tan asombrosa analogía con la primera, al iniciarse la evolución grávida, pudiéndose considerar como un adenoma temporario, encargado de producir colesterina.

La dosificación de las bilis grávidas muestra la intensidad de la formación: al paso que una bilis normal tiene unos $1,15=1,60$ de colesterina Mc Nee, en exámenes varias veces controlados comprueba un $6,7 \%$.

Otro factor enunciado por Bar es el metabolismo del hierro. La intensa destrucción de hematíes al nivel de la placenta y glándula hepática libera el $\mathrm{Fe}$, provocando una sobrecarga pigmentaria, espesando y haciendo más constante la bilis que excluyendo infección, puede dar una litiasis aséptica.

Estos factores, de alta intensidad en los dos últimos meses del embarazo, hacen que la posibilidad de una crisis inminente se acentúe, y así sucede en todas las gradaciones de la escala: desde una simple gastralgia hasta las colecistitis supuradas (empiema vesicular) no rara entre nosotros, la que junto con el cólico hepático y la angiocolitis aguda forman el objeto de este estudio.

A veces, en mujeres de excelente desarrollo físico, de grandes defensas orgánicas los accidentes sólo se presentan en la primera semana del puerperio o aún más tarde, haciendo sospechar en el primer caso una infección puerperal, en el segundo la amenaza de una fiebre eruptiva.

En general el $90 \%$ de las colecistectomizadas han tenido hijos. (Huyguens Henday y Crie).

La irritación continua, agravada por el golpear de los miembros inferiores del feto, en un útero casi siempre en dextroposición (traumatismo mínimo, pero constante) sobre la cara inferior del hígado materno, da facilidad para que se desencadene una infección en un aparato digestivo de condiciones precarias. Esta situación se anuncia por ictericia, escalofríos y fiebre. 
A veces el trabajo del parto despierta la situación dolorosa de la vesícula biliar, y ésta es palpable en el III período. En general, al estallar el cólico se ve acelerarse el trabajo. Si hay toxemia (Greenhill) la tensión se ha visto elevarse hasta escapar la máxima por encima del límite superior del esfigmomanómetro, de acuerdo con nuestra experiencia.

Vignes y Bar han señalado la presencia de un síndrome gravídico que envuelve la vesícula biliar y el apéndice.

\section{EL PROBLEMA DIAGNOSTICO}

En las crisis típicas $(2 / 5$ de los casos) el diagnóstico es evidente.

En las formas atípicas hay que tener en cuenta:

La intensidad sintomática.

La topografía del foco.

La naturaleza del terreno.

Con un cuadro clínico engañoso se hace incierto el diagnóstico. Deben observarse y recordarse:

La noción de la frecuencia de la colecistitis en nuestras mujeres en edad de actividad sexual y por ende en las gestantes.

Pensar en la edad y las condiciones del síndrome abdominal, alto, derecho y con antecedentes.

Pensar, aún en las formas más atípicas en la casi totalidad de las presentaciones de vértice, con dextroversión uterina que crea espinas irritativas en la región de Chauffard.

En los casos agudos (colecistitis supurada, empiema, al término) los dolores adquieren a veces una intensidad intolerable que requiere pronta sedación.

Cuando el trabajo se desencadena, éste es singularmente rápido, y se debe tener en cuenta dar una generosa profusión de oxígeno y de vitamina B-15, auncuando no hay un acuerdo total sobre esto último. 
El dolor sube al hombro derecho de ordinario. Se encuentran tres casos en esta situación:

a) Colecistitis aguda sin peritonitis,

b) Colecistitis aguda con participación peritoneal progresiva limitada,

c) Colecistitis aguda con peritonitis generalizada.

Esta es una clasificación general, que apenas serviría de orientación. El dolor del hombro derecho se borra para hacerse difuso en el abdomen, y la enferma, en el trabajo, excitada por el sufrimiento no halla alivio. Las contracciones, supeditadas al cólico pasan en general desapercibidas. Aquí no hay ni reposo, ni hielo que atenúen el dolor, exagerado por la vecindad del fondo uterino en trabajo.

En los casos supurados (Forma supurada recidivante de Hartmann) que han venido agravándose paulatinamente, pueden perforarse, siendo imprevisible esta evolución.

Se encuentra una forma muy especial que plantea graves dificultades diagnósticas y "que es la forma subictérica de Fiessinger".

Después de uno o varios brotes ha venido una crisis más violenta, agravada por la llegada del fondo uterino a la región vesicular. Es el cuadro de una obstrucción coledociana, sin grande escalofrío y sin real repercusión sobre el estado general. Esta benignidad relativa orienta el diagnóstico, que será seguido de confusión porque la ictericia se borra en dos semanas, baja la temperatura, pero la vesícula biliar ni retrocede, ni disminuye su sensibilidad.

Se han descrito colecistitis agudas no litiásicas, pero no se han hallado en la gestante.

El diagnóstico no es difícil, pero la situación vesicular prima sobre la obstétrica, agravándose cuando hay reacción local peritoneal: la temperatura oscila entre 38,5 y $40^{\circ} \mathrm{C}$. irregular unas 
veces, sostenida otras, el hipocondrio entero se hace pulsátil, la cara se excava, la lengua se hace profundamente saburral, indicios todos de infección grave.

El examen minucioso debe ir al abdomen, aquí ocupado por el útero en casi su totalidad, lo que dificulta la afirmación de la existencia de una lesión peritoneal. Un análisis pormenorizado y casi individual de los síntomas, una estrecha y atenta investigación conducirá el tratamiento y hará mermar el período de evolución.

La respiración es corta superficial, con reacción defensiva en las cercanías de la vesícula, para evitar su roce con un útero en trabajo. A veces llega hasta la inmovilidad del hemitórax inferior derecho y aún el hemiabdomen derecho, aunque estén levantados, se paralizan.

Las contracciones uterinas, sin característico dolor obstétrico lo dan en la cintura, y exquisito hacia la región vesicular.

La reacción peritoneal puede llegar a dar adherencias epiploicas o aún intestinales con empastamiento que se endurece a la contracción uterina. La defensa muscular es variable y se puede suavizar por la aplicación de hielo.

Se presenta: leucocitosis con polinucleosis, deficiencia hepática global, orinas escasas, ricas en albúmina y urobilina.

En una de las historias presentadas adelante, no se tuvo conocimiento exacto de la evolución, dada la mentalidad de la paciente, quien fue traída al servicio dos o tres horas después de su parto en plena reacción peritoneal. La más minuciosa de las exploraciones a cielo abierto no permitió encontrar la solución de continuidad por parte alguna y con grande asombro nuestro, el lavado del peritoneo, eliminando la bilis que empañaba asas y demás vísceras abdominales, con suero fisiológico simple, tibio, trajo una rápida recuperación

Hecho el diagnóstico de la combinación COLECISTITIS AGUDA-PARTO, no queda otro camino que la observación armada, y muy armada, aunque los aspectos ruidosamente clínicos no trai- 
gan la gravedad que parecen tener. Nosotros, con los oscuros conceptos de los autores consultados indicamos el apaciguamiento del drama abdominal angiopático. Sin embargo, encontramos en los casos presentados, que el tratamiento de sedación analgésica y antiespasmódica hubiera servido de mordiente dada su analogía en respuesta obstétrica a ia de una inducción hecha a base de drogas sedantes (demerol, escopolamina, largactil) libertando al parecer el sistema nervioso autónomo del útero, se le ha dejado en plena independencia para energizarse y contraerse por su cuenta, expeliendo fácilmente el producto de la concepción con un mínimo cortejo de dolores.

Parece que los metámeros dérmicos están paralizados y así se podría aprobar la afirmación de un artículo reciente en el que se dice que por medio de una campana neumática se elimina la porción parieto-abdominal en lo que se refiere al trabajo, con grande aceleración del mecanismo uterino independiente.

Resumiendo: en medio del auge del síndrome colangiopático, apenas se inicia una medicación analgésica o antiespasmódica, se puede aliviar generalmente el cortejo doloroso, desencadenándose un trabajo prácticamente indoloro y acelerado.

Entonces los grandes lineamientos de la conducta terapéutica llevan a buscar, por los medios apropiados, la analgesia de la sección dolorosa, con inmovilidad de la pared, aunque a su vez, por su propia cuenta el ganglio de Frankenhauser apresura -en autodefensa- la salida del feto para que el organismo pueda dedicar todas sus energías a vencer la colecistitis.

\section{PRONOSTICO}

Imposible por nuestra parte de fijarlo solamente con la presentación de tres casos.

En lo que a nosotros toca, pensamos que el pronóstico es bueno, dando los cuidados más estrictos a la enferma, y no descuidando ninguno de los factores que podrían ser causa de posible agravación. 


\section{TRATAMIENTO}

a) Médico

Como medida de urgencia, calmar el dolor.

No están contraindicados los opiáceos puesto que ya la lesión se ha caracterizado y localizado, y no va a ocultar - como en un clásico abdomen agudo- el drama peritoneal generalizado.

No hemos visto contractura generalizada (vientre de madera) ni una defensa difusa, sino más bien una sensibilidad exquisita local, a más de la presencia muchas veces apreciable con el solo cambio de ángulo visual de una tumoración bajo el reborde costal derecho. Polamidón, buscapina intravenosa, aminofilina, novalgina, demerol son los más efectivos sin que aumente el efecto nauseoso, tan notable digamos, en el pantopón.

Exceientes las aplicaciones térmicas, de preferencia secas como la cobija eléctrica; hay individuos que se benefician mejor con el hielo.

Levantamiento del estado general en una enferma deprimida. Anatensol, lucidol, clansed, son preferibles a los tranquilizantes ataráxicos.

Hidratación. Dextrosa al $5 \%$ con clorpromazina intravenosa darán alivio marcado.

Evacuación intestinal suave; 200 gramos de aceite de oliva, en caso de retardo excesivo en la marcha transintestinal, ayudados por un supositorio evacuante.

\section{b) Quirúrgico}

Con todos los requisitos apropiados, previa medicación intensa y delicada, sería la colecistotomía, abocando a la piel el fondo de la vesícula biliar.

En el caso relatado adelante, de una peritonitis biliar, se hizo un aseo peritoneal completo, dejando drenaje para quitar en 
cuanto acabara el flujo biliar. Allí no fue posible encontrar la solución de continuidad, ni el estado de la paciente permitía mayores escarceos quirúrgicos.

\section{HISTORIAS CLINICAS}

A. de M. Historia del I.C.S.S. No 345643 . - 33 años de edad, grávida 5 para 4. Llega al servicio con su ciclo de gestación cumplido en el día siguiente del calculado para el parto. Tensión arterial de 10-6. Conjuntivas subictéricas. Pulso 90. Respiraciones 40. Disnea que parece corresponder a la de su trabajo de parto. Hace cuatro horas que - según ella - ha iniciado un trabajo, el que encuentra absolutamente distinto del de sus otros partos. con sensación de que va a estallar la porción inferior del tórax, con irradiación al hombro derecho y estado de verdadero "stress".

Indagando antecedentes, esta señora ha sufrido con frecuencia de cólicos hepáticos. En todos sus embarazos se ha exacerbado el mal vesicular. Durante eze tiempo no puede alimentarse sino de frutas, ensaladas, caldos de carne, galletas. La menor separación de este régimen desencadena el drama vesicular.

Altura uterina de $33 \mathrm{cms}$. Soplo placentario en el cuadrante superior uterno izquierdo. A la mitad de la línea umbílico espina ilíaca superior izquierda suenan bien timbrados ruidos fetales a 150-minuto. Vértice, dorso a la izquierda; pequeñas partes móviles en la vecindad del colecisto. Bajo el reborde costal se aprecia una tumoración del tamaño del puño, fluctuante, atrozmente sensible.

T. V. Cuello borrado con una dilatación que da paso a un dedo el orificio externo; el interno está cerrado. Presentación de vértice, fija totalmente cubierta por un seomento inferior muy delgado.

No se aprecian contracciones uterinas. Dolor alto, irradiado al hombro derecho, hiperestesia general del hipocondrio derecho.

Se deja en observación. Dado su estado, con facies ligeramente desencajada, con nariz afilada, ligera hipertermia se pasa por vía endovenosa una dextrosa al $5 \%$ con 250 mgrs. de tetraciclina en forma lenta. Se dan 200 gramos de aceite de olivas por vía oral, un supositorio de atrinal y una bolsa pequeña de hielo sobre la vesícula biliar.

Fórmula hemática con 20.000 leucocitos, intensa polinucleosis. La temperatura asciende en la primera tarde a $40^{\circ} \mathrm{C}$, baja a la media noche a $38^{\circ} \mathrm{C}$. se mantiene en una línea más o menos horizontal.

Aquí de la vacilación. Se opera la enferma o nó? Hay expectativa. Pero en 48 horas los síntomas generales retroceden. Pasa el dolor, pero tampoco se ha establecido el trabajo del parto. 
Vol. XII

En vista de que se continúa palpando una gran vesícula en la que se supone constituído un empiema, los cirujanos resuelven operar.

A los dos días de calma, se premedica y se pasa al quirófano. Sin que nada lo hiciera prever, en la camilla que la conduce estalla la bolsa de las aguas, iniciándose un rápido trabajo. Claras contracciones dolorosas se aprecian durante el período preoperatorio (sonda, lavado al éter y pintura de la pared abdominal con mertiolato).

Se suspende la marcha de la ceremonia operatoria, se traslada a la sala de partos y en menos de una hora da a luz un feto de sexo masculino de 3.500 gramos de peso.

Puerperio excelente. Vesícula grande, pero su sensibilidad se ha atenuado. Se espera que termine el puerperio para intervenir.

\section{N. L. del Servicio de Maternidad de las Fuerzas Armadas.}

Grávida IV. Da a luz un feto femenino en el carro que la lleva a la Clínica a donde llega en estado de shock. Hemorragia muy moderada, pero hay un dolor de sensación de puñalada, en el hipocondrio derecho que la sumió en este estado.

Tensión arterial 7-2. 150 pulsaciones depresibles, débiles. Temperatura bucal 36. Cubierta de sudor frío; vientre fláccido de multípara en el que resalta un globo uterino de $25 \mathrm{cc}$. de altura. Sensibilidad exquisita por todo el abdomen. Extremidades frías. 35 respiraciones anhelantes.

Esta enferma cuenta que durante todo su embarazo ha padecido de cóli$\cos$ hepáticos y que cada uno de ellos ha sido un episodio realmente dramático.

Su trabajo de parto aparentemente comenzó en la mañana unas cuatro horas antes de este examen. Inmediatamente comenzó sus preparativos para venir a hospitalizarse. Los dolores los califica de "raros". En el taxi rompió las membranas, tuvo la niña, y la placenta que parece completa. No sangró mucho pero el estado general es bastante malo.

Se lleva a la sala de cirugía y se prepara para una laparotomía exploradora: dextrosa al $10 \%$, calentamiento, Cedilanid intravenoso, dado un corazón rápido y débil, Veritol, Atropina y Fenergán.

Laparotomía mediana infraumbilical. Al abrir el peritoneo se hallan todos los órganos empapados en bilis. Se prolonga la incisión hacia arriba, afortunadamente la enferma no es obesa y permite una exploración muy detenida de la cara inferior del hígado y de todo el aparato biliar que se irriga con suero tibio. Por ninguna parte se descubre la solución de continuidad que ha dejado escapar toda esta bilis. 
Se continúa la dextrosa con 250 mgms. de tetraciclina; 500 cc. de sangre. Cierre en planos de la pared, dejando en la parte inferior de la herida un dren por donde durante 24 horas escurre suero teñido de bilis, luego se seca.

Recuperación completa y puerperio excelente.

O. T. de 25 años de edad. Primípara.

Llamada de urgencia el día calculado para su parto.

Desde media noche tiene un dolor que parece absolutamente diferente de los que su madre le ha indicado como sintomático de parto. Le invade la mitad inferior del hemitórax derecho, inmovilizado en defensa, con una respiración absolutamente superficial; el dolor se irradia al hombro y se exacerba con cualquier movimiento.

En la clínica protesta por el examen vaginal que se le hace, ya que el máximo de dolor está en el hombro derecho. Se encuentra sin embargo, un cuello ya completamente borrado, con unos 4 cc. de dilatación y membranas intactas. Presentación profundamente encajada en OIIA. Ruidos fetales buenos, dorso hacia la izquierda, altura uterina de $33 \mathrm{cms}$.

Tensión arterial de 10-6. Pulso de 120 . Temperatura de $37,8^{\circ} \mathrm{C}$. Conjuntivas subictéricas. El dolor se hace difuso en la parte superior del abdomen.

No ha tenido cólicos hepáticos. Pero desde que quedó embarazada ha sufrido intensas molestias: vómitos intensos desde el primer mes, fastidio abdominal intenso, odio al marido, accesos de ira. Mejoró algo por tratamiento hipnótico. Todo lo cual además de frecuentes accesos de diarrea blanquecina, se completa con la sensación de una "bola" al lado derecho, por encima del ombligo.

Allí bajo el reborde costal se puede, no obstante la solidez de un excelente y espeso tejido celular subcutáneo, como el de toda primigestante bien cuidada se percibe el fondo fluctuante de una vesícula biliar crecida y sensible.

Se presenta y se intensifica un cólico hepático. Se acude a la medicación antiespasmódica y analgésica máxima durante 48 horas en toda su plenitud. La temperatura oscila de mañana a tarde entre 37,5 y 39 . Ligeramente estabilizada y cedido el estado doloroso grave parece querer reiniciarse, pero antes de que se acuda a la analgesia, se inicia un trabajo a la velocidad de una inducción efectiva, expulsando un feto femenino de 3.570 gramos en cuarenta minutos. Episiotomía amplia, perineorrafia, retroceso de todos los fenómenos, tanto colangiopáticos como de puerperio normal.

\section{RESUMEN Y CONCLUSIONES}

Se exponen situaciones de colecistitis aguda, empiema vesicular y trabajo de parto, para cuya conducta no fue posible encon- 
trar orientación en la literatura consultada, adoptándose una conducta enteramente personal

A pesar de la gravedad aparente de los cuadros clínicos, el síndrome vesicular cedió con relativa facilidad, dando rapidez y forma sencilla a las situaciones obstétricas.

Desde el comienzo de la vigilancia prenatal debe iniciarse una investigación sobre el estado físico, funcional y calidad de la vesícula biliar.

Ante el estallido de una colecistitis aguda, de un empiema y de embarazo a término o trabajo, se debe dar preferencia terapéutica al sistema biliar, permitiendo el desarrollo espontáneo de los fenómenos obstétricos.

Desencadenado - como suele suceder- el trabajo que avanza con extraordinaria rapidez, aún en la primigestante, da lugar a eliminarle rápidamente y concretarse al aparato biliar. En Francia, EE. UU. y aún en Bogotá, con nuestra superespecialización, estas enfermas deberían trasladarse a servicios de medicina interna. En los pequeños hospitales de provincia se advierte que no es necesario el aislamiento para ellas, aún febricitantes y que si la madre tiene suficiente leche y desea suministrarla a su recién nacido puede hacerlo sin mayores inconvenientes.

\section{BIBLIOGRAFIA}

(1) Estadísticas de 1932-1942 del Instituto Materno Infantil. No publicadas.

(2) Estadística de 1947-1957 del Servicio de Maternidad en la Sanidad Militar (Familiares). No publicadas.

(3) Estadística de 1949-1959 en el ICSS. No publicada.

(4) BAR y BRINDEAU. 1940. Pratique de l'Art des Accouchements. París 1940. Vigot Freen.

(5) GREENHILL y DELEE. 1955. Principles and practice of Obstetrics. Uthea 1955.

(6) DEXEUS y FONT 1956. Obstetricia. Salvat Editores. Barcelona, 1952.

(7) MORAgUEST y BERNAT, Clínica Obstétrica. Ed. El Ateneo, 1948.

(8) CARL DAVIS. Obstetrics. W. F. Prior Company. Maryland, U.S.A. 